\title{
Topological search for the production of neutralinos and scalar particles
}

\section{OPAL Collaboration}

G. Alexander ${ }^{w}$, J. Allison ${ }^{p}$, N. Altekamp ${ }^{\mathrm{e}}$, K. Ametewee ${ }^{\mathrm{y}}$, K.J. Anderson ${ }^{\mathrm{i}}$, S. Anderson ${ }^{\ell}$, S. Arcelli ${ }^{\text {b }}$, S. Asai ${ }^{x}$, D. Axen ${ }^{\text {ac }}$, G. Azuelos ${ }^{\text {r,1 }}$, A.H. Ball ${ }^{\text {q }}$, E. Barberio ${ }^{2}$, R.J. Barlow ${ }^{p}$, R. Bartoldus ${ }^{c}$, J.R. Batley ${ }^{\mathrm{e}}$, G. Beaudoin ${ }^{\mathrm{r}}$, J. Bechtluft ${ }^{\mathrm{n}}$, C. Beeston ${ }^{\mathrm{p}}$, T. Behnke ${ }^{\mathrm{h}}$, A.N. Bell ${ }^{\text {a }}$, K.W. Bell ${ }^{\mathrm{t}}$, G. Bella ${ }^{\mathrm{w}}$, S. Bentvelsen ${ }^{\mathrm{h}}$, P. Berlich $^{\mathrm{j}}$, S. Bethke ${ }^{\mathrm{n}}$, O. Biebel ${ }^{\mathrm{n}}$, V. Blobel ${ }^{\text {h}}$, I.J. Bloodworth ${ }^{\mathrm{a}}$, J.E. Bloomer ${ }^{\mathrm{a}}$, P. Bock ${ }^{\mathrm{k}}$, H.M. Bosch ${ }^{\mathrm{k}}$, M. Boutemeur ${ }^{\mathrm{r}}$, B.T. Bouwens ${ }^{\ell}$, S. Braibant ${ }^{\ell}$, P. Bright-Thomas ${ }^{y}$, R.M. Brown', H.J. Burckhart ${ }^{\text {h }}$, C. Burgard ${ }^{\text {aa }}$, R. Bürgin ${ }^{j}$, P. Capiluppi ${ }^{\text {b }}$, R.K. Carnegie ${ }^{f}$, A.A. Carter ${ }^{\mathrm{m}}$, J.R. Carter ${ }^{\mathrm{e}}$, C.Y. Chang ${ }^{\text {q }}$, C. Charlesworth ${ }^{\mathrm{f}}$, D.G. Charlton ${ }^{\mathrm{a}, 2}$, D. Chrisman ${ }^{\mathrm{d}}$, S.L. $\mathrm{Chu}^{\mathrm{d}}$, P.E.L. Clarke ${ }^{o}$, I. Cohen ${ }^{\text {w }}$, J.E. Conboy ${ }^{o}$, O.C. Cooke ${ }^{\text {p }}$, M. Cuffiani ${ }^{b}$, S. Dado ${ }^{v}$, C. Dallapiccola ${ }^{\mathrm{q}}$, G.M. Dallavalle ${ }^{\mathrm{b}}$, C. Darling ${ }^{\mathrm{ae}}$, S. De Jong ${ }^{\ell}$, L.A. del Pozo ${ }^{\mathrm{h}}$, M.S. Dixit ${ }^{g}$, E. do Couto e Silva ${ }^{\ell}$, M. Doucet ${ }^{r}$, E. Duchovni ${ }^{z}$, G. Duckeck ${ }^{\mathrm{h}}$, I.P. Duerdoth ${ }^{p}$, J.E.G. Edwards ${ }^{p}$, P.G. Estabrooks ${ }^{f}$, H.G. Evans ${ }^{i}$, M. Evans ${ }^{\text {m }}$, F. Fabbri ${ }^{b}$, P. Fath ${ }^{k}$, F. Fiedler ${ }^{\ell}$, M. Fierro ${ }^{b}$, H.M. Fischer ${ }^{\text {c }}$, R. Folman ${ }^{z}$, D.G. Fong ${ }^{q}$, M. Foucher ${ }^{q}$, H. Fukui ${ }^{x}$, A. Fürtjes ${ }^{\text {h }}$, P. Gagnon ${ }^{g}$, A. Gaidot ${ }^{\text {u }}$ J.W. Gary ${ }^{\text {d }}$, J. Gascon ${ }^{\text {r }}$, S.M. Gascon-Shotkin ${ }^{q}$, N.I. Geddes ${ }^{\prime}$, C. Geich-Gimbel ${ }^{\text {c }, ~ S . W . ~ G e n s l e r ~}{ }^{i}$, F.X. Gentit ${ }^{u}$, T. Geralis ${ }^{t}$, G. Giacomelli ${ }^{b}$, P. Giacomelli ${ }^{\mathrm{d}}$, R. Giacomelli ${ }^{\mathrm{b}}$, V. Gibson ${ }^{\mathrm{e}}$, W.R. Gibson ${ }^{\mathrm{m}}$, D.M. Gingrich ${ }^{\text {ad,l }}$, J. Goldberg ${ }^{\text {v }}$, M.J. Goodrick ${ }^{\mathrm{e}}$, W. Gorn ${ }^{\mathrm{d}}$, C. Grandi ${ }^{\mathrm{b}}$, E. Gross ${ }^{\mathrm{z}}$, M. Gruwé ${ }^{h}$, C. Hajdu ${ }^{\text {af }}$, G.G. Hanson ${ }^{\ell}$, M. Hansroul ${ }^{\text {h }}$, M. Hapke ${ }^{m}$, C.K. Hargrove ${ }^{g}$, P.A. Hart ${ }^{\mathrm{i}}$, C. Hartmann ${ }^{\mathrm{c}}$, M. Hauschild ${ }^{\text {h }}$, C.M. Hawkes ${ }^{\mathrm{e}}$, R. Hawkings ${ }^{\mathrm{h}}$, R.J. Hemingway ${ }^{\text {, }}$, G. Herten ${ }^{j}$, R.D. Heuer ${ }^{\text {h }}$, M.D. Hildreth ${ }^{\text {h }}$, J.C. Hill ${ }^{\mathrm{e}}$, S.J. Hillier ${ }^{\mathrm{a}}$, T. Hilse ${ }^{\mathrm{i}}$, P.R. Hobson ${ }^{y}$, R.J. Homer ${ }^{\mathrm{a}}$, A.K. Honma ${ }^{\text {ab, }}{ }^{\text {, D. Horváth }}{ }^{\mathrm{af}, 3}$, R. Howard ${ }^{\mathrm{ac}}$, R.E. Hughes-Jones ${ }^{p}$, D.E. Hutchcroft ${ }^{\mathrm{e}}$, P. Igo-Kemenes ${ }^{\mathrm{k}}$, D.C. Imrie ${ }^{\mathrm{y}}$, M.R. Ingram ${ }^{\mathrm{p}}$, A. Jawahery ${ }^{q}$, P.W. Jeffreys ${ }^{t}$, H. Jeremie ${ }^{r}$, M. Jimack ${ }^{a}$, A. Joly ${ }^{r}$, G. Jones ${ }^{p}$, M. Jones ${ }^{f}$, R.W.L. Jones ${ }^{\text {h }}$, U. Jost ${ }^{k}$, P. Jovanovic ${ }^{\text {a }}$, J. Kanzaki ${ }^{x}$, D. Karlen ${ }^{\text {f }}$, T. Kawamoto ${ }^{x}$,

R.K. Keeler ${ }^{\text {ab }}$, R.G. Kellogg ${ }^{\text {q }}$, B.W. Kennedy ${ }^{t}$, J. King ${ }^{\mathrm{m}}$, J. Kirk ${ }^{\text {ac }}$, S. Kluth ${ }^{\mathrm{h}}$,

T. Kobayashi ${ }^{x}$, M. Kobel ${ }^{\text {j}}$, D.S. Koetke ${ }^{\text {f }}$, T.P. Kokott ${ }^{\text {c }}$, S. Komamiya ${ }^{x}$, R. Kowalewski ${ }^{\text {h}}$,

T. Kress ${ }^{k}$, P. Krieger ${ }^{f}$, J. von Krogh ${ }^{k}$, P. Kyberd ${ }^{\mathrm{m}}$, G.D. Lafferty ${ }^{\mathrm{p}}$, H. Lafoux ${ }^{\mathrm{u}}$, R. Lahmann ${ }^{\text {q, W.P. Lai }}{ }^{\mathrm{s}}$, D. Lanske ${ }^{\mathrm{n}}$, J. Lauber ${ }^{\mathrm{o}}$, J.G. Layter ${ }^{\mathrm{d}}$, A.M. Lee ${ }^{\text {ae }}$, E. Lefebvre ${ }^{\mathrm{r}}$, 
D. Lellouch ${ }^{2}$, J. Letts ${ }^{b}$, L. Levinson ${ }^{2}$, C. Lewis ${ }^{\circ}$, S.L. Lloyd ${ }^{m}$, F.K. Loebinger ${ }^{p}$, G.D. Long ${ }^{\mathrm{q}}$, B. Lorazo ${ }^{\mathrm{r}}$, M.J. Losty ${ }^{\mathrm{g}}$, J. Ludwig ${ }^{\mathrm{j}}$, A. Luig $^{\mathrm{j}}$, A. Malik ${ }^{\mathrm{u}}$, M. Mannelli ${ }^{\mathrm{h}}$, S. Marcellini ${ }^{\text {b }}$, C. Markus ${ }^{\text {c }}$, A.J. Martin ${ }^{\mathrm{m}}$, J.P. Martin ${ }^{\mathrm{r}}$, G. Martinez ${ }^{\mathrm{q}}$, T. Mashimo ${ }^{\mathrm{x}}$, W. Matthews ${ }^{y}$, P. Mättig ${ }^{c}$, W.J. McDonald ${ }^{\text {ad }}$, J. McKenna ${ }^{\text {ac }}$, E.A. Mckigney ${ }^{\circ}$, T.J. McMahon ${ }^{\text {a }}$, A.I. McNab ${ }^{\mathrm{m}}$, F. Meijers ${ }^{\text {h}}$, S. Menke ${ }^{\text {c }}$, F.S. Merritt ${ }^{\mathrm{i}}$, H. Mes ${ }^{\mathrm{g}}$, J. Meyer ${ }^{\text {aa }}$, A. Michelini ${ }^{\text {h}}$, G. Mikenberg ${ }^{\text {z }}$, D.J. Miller ${ }^{\circ}$, R. $\operatorname{Mir}^{2}$, W. Mohr ${ }^{j}$, A. Montanari ${ }^{b}$, T. Mori ${ }^{x}$, M. Morii ${ }^{x}$, U. Müller $^{c}$, B. Nellen ${ }^{c}$, B. Nijjhar ${ }^{p}$, R. Nisius ${ }^{h}$, S.W. O'Neale ${ }^{\text {a }}$, F.G. Oakham ${ }^{g}$, F. Odorici ${ }^{\text {b }}$, H.O. Ogren ${ }^{\ell}$, T. Omori ${ }^{x}$, M.J. Oreglia ${ }^{i}$, S. Orito ${ }^{\mathrm{x}}$, M. Palazzo ${ }^{\mathrm{b}}$, J. Pálinkás ${ }^{\mathrm{ag}, 4}$, J.P. Pansart ${ }^{\mathrm{u}}$, G. Pásztor ${ }^{\text {af }}$, J.R. Pater ${ }^{\mathrm{p}}$, G.N. Patrick ${ }^{\mathrm{t}}$, M.J. Pearce ${ }^{\mathrm{a}}$, S. Petzold ${ }^{\text {aa }}$, J.E. Pilcher ${ }^{\mathrm{i}}$, J. Pinfold ${ }^{\text {ad }}$, D.E. Plane ${ }^{\mathrm{h}}$, P. Poffenberger ${ }^{a b}$, B. Poli ${ }^{b}$, A. Posthaus ${ }^{c}$, H. Przysiezniak ${ }^{\text {ad }}$, D.L. Rees ${ }^{a}$, D. Rigby ${ }^{a}$, M.G. Rison ${ }^{\mathrm{e}}$, S.A. Robins ${ }^{\mathrm{m}}$, N. Rodning ${ }^{\text {ad }}$, J.M. Roney ${ }^{\text {ab }}$, A. Rooke ${ }^{\mathrm{o}}$, E. Ros ${ }^{\mathrm{h}}$, A.M. Rossi ${ }^{\text {b }}$, M. Rosvick ${ }^{\text {ab }}$, P. Routenburg ad, Y. Rozen ${ }^{\text {h }}$, K. Runge ${ }^{\mathrm{j}}$, O. Runolfsson ${ }^{\mathrm{h}}$, D.R. Rust ${ }^{\ell}$, R. Rylko ${ }^{y}$, E.K.G. Sarkisyan ${ }^{\text {w }}$, M. Sasaki ${ }^{x}$, C. Sbarra ${ }^{b}$, A.D. Schaile ${ }^{\text {h,5 }}$, O. Schaile ${ }^{j}$, F. Scharf ${ }^{\text {c }}$, P. Scharff-Hansen ${ }^{\text {h }}$, P. Schenk ${ }^{d}$, B. Schmitt ${ }^{c}$, M. Schröder ${ }^{\text {h }}$, H.C. Schultz-Coulon ${ }^{j}$, M. Schulz ${ }^{h}$, P. Schütz ${ }^{c}$, J. Schwiening ${ }^{c}$, W.G. Scott ${ }^{t}$, T.G. Shears ${ }^{p}$, B.C. Shen ${ }^{\mathrm{d}}$, C.H. Shepherd-Themistocleous ${ }^{\text {aa }}$, P. Sherwood ${ }^{\circ}$, G.P. Siroli ${ }^{b}$, A. Sittler $^{\text {aa }}$, A. Skillman ${ }^{\circ}$, A. Skuja ${ }^{q}$, A.M. Smith ${ }^{\text {h}}$, T.J. Smith ${ }^{\text {ab }}$, G.A. Snow ${ }^{q}$, R. Sobie ${ }^{\text {ab }}$, S. Söldner-Rembold ${ }^{j}$, R.W. Springer ${ }^{\text {ad }}$, M. Sproston ${ }^{t}$, A. Stahl ${ }^{c}$, M. Starks ${ }^{\ell}$, K. Stephens ${ }^{p}$, J. Steuerer ${ }^{\text {aa }}$, B. Stockhausen ${ }^{c}$, D. Strom ${ }^{\text {s }}$, F. Strumia ${ }^{\text {h }}$, P. Szymanski ${ }^{t}$, R. Tafirout ${ }^{r}$, H. Takeda ${ }^{\mathrm{x}}$, P. Taras ${ }^{\mathrm{r}}$, S. Tarem ${ }^{\mathrm{v}}$, M. Tecchio ${ }^{\mathrm{h}}$, N. Tesch ${ }^{\mathrm{c}}$, M. Thiergen ${ }^{\mathrm{j}}$, M.A. Thomson ${ }^{\mathrm{h}}$, E. von Törne ${ }^{\mathrm{c}}$, S. Towers ${ }^{\mathrm{f}}$, M. Tscheulin ${ }^{\mathrm{j}}$, E. Tsur ${ }^{\mathrm{w}}$, A.S. Turcot ${ }^{\mathrm{i}}$, M.F. Turner-Watson ${ }^{\mathrm{h}}$, P. Utzat ${ }^{k}$, R. Van Kooten ${ }^{\ell}$, G. Vasseur ${ }^{u}, M$. Verzocchi $^{j}$, P. Vikas ${ }^{r}, M$. Vincter $^{\text {ab }}$, E.H. Vokurka ${ }^{\text {p}}$, F. Wäckerle ${ }^{\mathrm{j}}$, A. Wagner ${ }^{\text {aa }}$, C.P. Ward ${ }^{\mathrm{e}}$, D.R. Ward ${ }^{\mathrm{e}}$, J.J. Ward ${ }^{\mathrm{o}}$, P.M. Watkins ${ }^{a}$, A.T. Watson ${ }^{a}$, N.K. Watson ${ }^{\mathrm{g}}$, P. Weber ${ }^{\mathrm{f}}$, P.S. Wells ${ }^{\mathrm{h}}$, N. Wermes ${ }^{\mathrm{c}}$, J.S. White ${ }^{a b}$, B. Wilkens ${ }^{\mathrm{j}}$, G.W. Wilson ${ }^{\text {aa }}$, J.A. Wilson ${ }^{\mathrm{a}}$, T. Wlodek ${ }^{\mathrm{z}}$, G. Wolf ${ }^{\mathrm{z}}$, S. Wotton ${ }^{k}$, T.R. Wyatt ${ }^{p}$, S. Xella ${ }^{b}$, S. Yamashita ${ }^{x}$, G. Yekutieli $^{z}$, K. Yoshimura ${ }^{x}$, V. Zacek ${ }^{\mathrm{r}}$

School of Physics and Space Research, University of Birmingham, Birmingham B/5 2TT, UK

' Dipartimento di Fisica dell' Università di Bologna and INFN, I-40126 Bologna, Italy c Physikalisches Institut, Universität Bonn, D-53115 Bonn, Germany

d Department of Physics, University of California, Riverside CA 92521, USA c Cavendish Laboratory, Cambridge CB3 OHE, UK

Ottawa-Carleton Institute for Physics, Department of Physics, Carleton University, Ottawa. Ontario KIS 5B6, Canada

\& Centre for Research in Particle Physics, Carleton University, Ottawa, Ontario KIS 5B6, Canada

${ }^{\mathrm{h}}$ CERN. European Organisation for Particle Physics, CH-1211 Geneva 23, Switzerland

i Enrico Fermi Institute and Department of Physics, University of Chicago, Chicago IL 60637. USA

i Fakultät für Physik, Albert Ludwigs Universität, D-79104 Freiburg, Germany

k Physikalisches Institut. Universität Heidelberg, D-69120 Heidelberg, Germany

'Indiana University, Department of Physics, Swain Hall West 117, Bloomington IN 47405. USA

${ }^{m}$ Queen Mary and Westfield College, University of London, London El 4NS, UK

"Technische Hochschule Aachen, III Physikalisches Institut, Sommerfeldstrasse 26-28, D-52056 Aachen, Germany "University College London, London WCIE 6BT, UK

P Department of Physics, Schuster Laboratory, The University, Manchester MI3 9PL, UK

${ }^{4}$ Department of Physics, University of Maryland, College Park, MD 20742, USA 


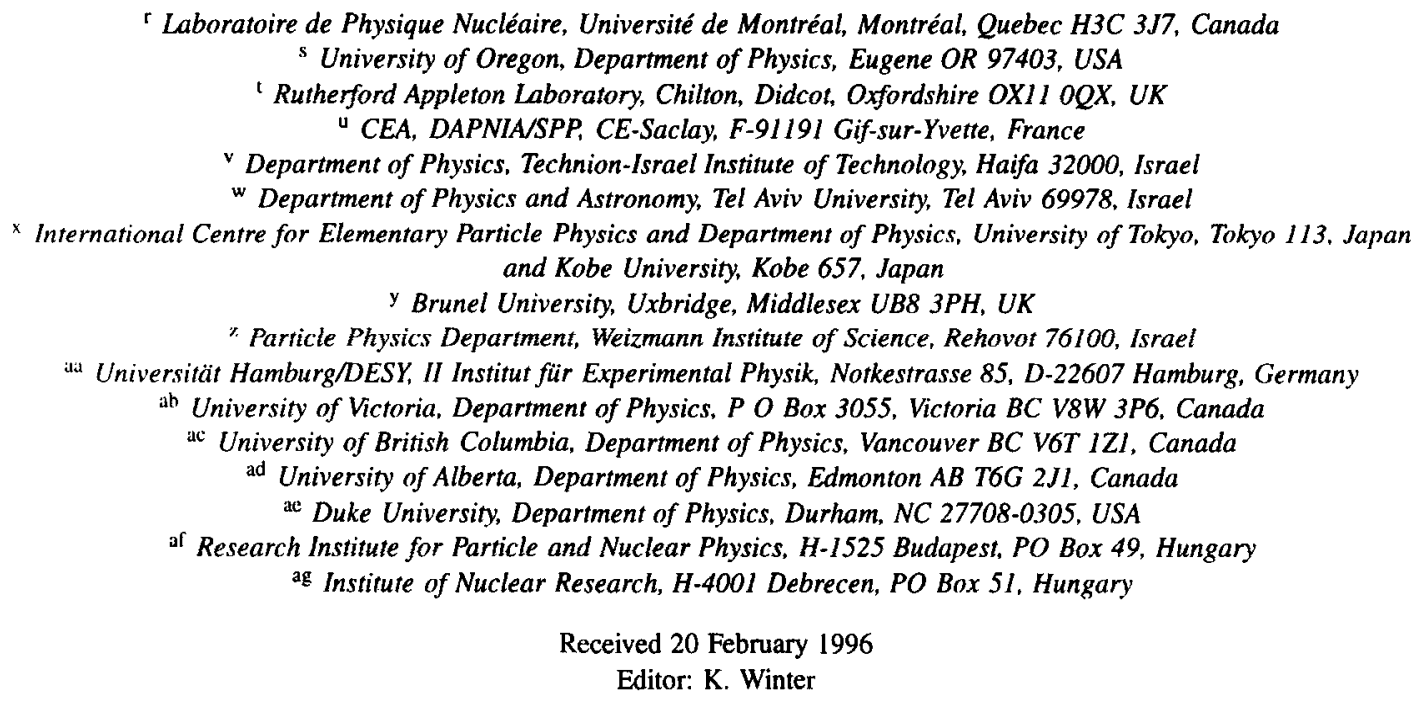

\begin{abstract}
A search for scalar particles and neutralinos such as those predicted by supersymmetric models has been performed using a data sample of 4.4 million hadronic $Z^{0}$ decays recorded by the OPAL detector at the $\mathrm{e}^{+} \mathrm{e}^{-}$collider LEP. The production of such particles typically leads to event topologies consisting of an acoplanar pair of jets, or of a mono-jet, accompanied by sizeable missing energy owing to neutrinos and other undetectable neutral particles. Limits are obtained, at the $95 \%$ confidence level, on the masses and production rates of scalar particles produced in association with the $\mathbf{Z}^{0}$. Limits are also placed on neutralino production, for which an additional possible signature is also studied, events containing a single observed photon. Within the Minimal Supersymmetric Standard Model, the mass of the lightest neutralino is found to be larger than $12.5 \mathrm{GeV} / c^{2}$ at $95 \%$ C.L., provided that $\tan \beta$ is larger than 1.5 .
\end{abstract}

\title{
1. Introduction
}

The data collected with the OPAL detector during the years 1990-1995, amounting to approximately 4.4 million hadronic $Z^{0}$ decays, were analysed in order to look for events with large missing energy, containing either a single jet ("mono-jet") or an "acoplanar" pair of jets, i.e. jets which are not back-to-back in the transverse plane. Such topologies are characteristic of several interesting processes involving the production of scalar or supersymmetric particles. The events observed with such topologies are compatible with expected backgrounds from standard processes, and our results may be used to place limits on the production of a scalar particle produced in association with a virtual $Z^{0}$ boson $\left(Z^{*}\right)$, where the scalar decays either into quarks or into invisible neutral particles. Limits are also placed on neutralino production, for which data on single photon production and on the $Z^{0}$ width are also used.

\footnotetext{
${ }^{1}$ And at TRIUMF, Vancouver, Canada V6T 2 A3

2 And Royal Society University Research Fellow

${ }^{3}$ And Institute of Nuclear Research, Debrecen, Hungary

${ }^{+}$And Depart of Experimental Physics, Lajos Kossuth University, Debrecen, Hungary

${ }^{5}$ And Ludwig-Maximilians-Universität, München, Germany
} 
In the Standard Model [1] (SM) of electroweak interactions, a complex doublet of scalar fields is introduced in order to endow the $\mathrm{W}^{ \pm}$and $\mathrm{Z}^{0}$ gauge bosons with mass [2]. This leads to the prediction that a scalar Higgs boson $\left(\mathrm{H}^{0}\right)$ should exist, of unspecified mass, but well-defined couplings. Any extension of the Standard Model with two or more complex doublets leads to additional scalar Higgs particles. The lightest of these particles would typically couple to the $\mathrm{Z}^{0}$ with a smaller coupling than the SM Higgs. In a large class of models it decays in a manner similar to that of a SM Higgs [3], mostly to a pair of heavy quarks. Such scalars should be produced in association with a virtual $Z^{*}$ which decays to neutrinos with branching fraction $\sim 20 \%$. A clear signature for scalar production would therefore be hadronic events with unbalanced momentum and large missing energy.

A similar topology may occur if the $Z^{0}$ boson decays into a pair of neutralinos, which are predicted in supersymmetric (SUSY) theories [4]. The fermionic partners of the $\gamma$ (photinos), the $Z^{0}$ boson (zinos), and the neutral Higgs boson (neutral Higgsinos) mix to form mass eigenstates $\left(\tilde{\chi}_{i}^{0}\right)$ called neutralinos. The sign of the mass cigenvalues corresponds to the product of their charge-conjugation and parity (CP) quantum numbers. In the present analysis the lightest neutralino $\tilde{\chi}_{1}^{0}$ is assumed to be the lightest supersymmetric particle and is therefore stable and invisible if R-parity [5] is conserved. The pair production of $\tilde{\chi}_{2}^{0} \tilde{\chi}_{1}^{0}$ or $\tilde{\chi}_{2}^{0} \tilde{\chi}_{2}^{0}$, where $\tilde{\chi}_{2}^{0}$ is the second lightest neutralino, followed by the decay of $\tilde{\chi}_{2}^{0}$ into $\tilde{\chi}_{1}^{0} Z^{*}$, with $Z^{*} \rightarrow q \bar{q}$, leads to the topology of an acoplanar pair of jets, or a mono-jet. Neutralino pairs $\left(\tilde{\chi}_{i}^{0} \tilde{\chi}_{j}^{0}\right)$ can be produced through $s$-channel $Z^{0}$ boson decay or by $t$-channel selectron $(\tilde{e})$ exchange. The latter diagram gives a significant constructive contribution to the production rate if the selectron is light. However, in that case, the decay of $\tilde{X}_{2}^{0}$ to $\bar{\nu} \tilde{\nu}$ may dominate for certain values of the SUSY parameters, leading to unobserved final states.

The simplest supersymmetric extension of the SM is the Minimal Supersymmetric Standard Model [6] (MSSM), which involves the addition of only one complex doublet of scalar fields to the SM. In the MSSM there might be a large branching ratio for the decay $\tilde{\chi}_{2}^{0} \rightarrow \tilde{\chi}_{1}^{0} \gamma$ [7]. Therefore events in which a single photon is detected are also included in the present study.

Finally we consider the case of an invisible scalar which couples to the $Z^{0}$ with some fraction of the coupling of the SM Higgs boson, produced in association with a virtual $Z^{*}$. Such a scenario could occur, for example, in the MSSM, where the light scalar Higgs might decay to a pair of invisible neutralinos. Invisible scalar particles also appear in other models, such as Majoron models ([8-11]). Some of these models are suggested for the generation of the cosmological baryon asymmetry at the electroweak scale [12]. In all of these models the scalar boson could decay predominantly into invisible majorons [10-13]. In order to search for invisible scalars produced in association with a virtual $\mathrm{Z}^{*}$, one can exploit the $70 \%$ decay branching fraction of the $\mathrm{Z}^{0}$ boson to a quark pair. The experimental signature is therefore essentially the same as for a scalar decaying to quarks recoiling against a $Z^{*}$ decaying to neutrinos.

In this paper we report on a direct search for events with acoplanar jets, mono-jets or single photons, using the OPAL detector at centre-of-mass energies around the $Z^{0}$ mass. In Section 2 we outline the detector, and the data and Monte Carlo samples used for the analysis. In Section 3 the analysis procedure is described, in Section 4 the efficiencies and systematic errors are discussed, and in Section 5 the results are interpreted in terms of limits on particle production rates within the framework of some of the models mentioned above.

\section{The OPAL detector, data selection and simulation}

The OPAL detector is described in detail in Ref. [14]. It is a multipurpose apparatus having nearly complcte solid angle coverage. The central detector consists of a system of tracking chambers providing charged particle tracking over $96 \%$ of the full solid angle ${ }^{6}$ inside a $0.435 \mathrm{~T}$ solenoidal magnetic field. A lead-glass electromagnetic calorimeter located outside the magnet coil covers the full azimuthal range with excellent hermeticity in

\footnotetext{
"A right-handed coordinate system is adopted, where the $x$-axis points to the centre of the LEP ring, and positive $z$ is along the electron beam direction. The angles $\theta$ and $\phi$ are the polar and azimuthal angles, respectively.
} 
the polar angle range $|\cos \theta|<0.82$ for the barrel region and $0.81<|\cos \theta|<0.984$ for the endcap region. The magnet return yoke is instrumented for hadron calorimetry, and consists of barrel and endcap sections along with pole tips that together cover the region $|\cos \theta|<0.99$. Calorimeters close to the beam axis measure the luminosity using small angle Bhabha scattering events and complete the geometrical acceptance down to $26 \mathrm{mrad}$. These include the forward detectors (FD) which are lead-scintillator sandwich calorimeters and at smaller angles, silicon-tungsten calorimeters (SW) [15] located on both sides of the interaction point.

The data sample used for the present analysis includes about 4.4 million hadronic $Z^{0}$ decays collected at energies around the $Z^{0}$ peak, corresponding to a total integrated luminosity of approximately $160 \mathrm{pb}^{-1}$. Standard quality requirements on tracks and energy clusters in the calorimeters were imposed, as described in Ref. [16]. In brief, the tracks used in this study were required to originate at the interaction point, to have greater than some minimum number of hits in the main tracking chamber, and to have a transverse momentum with respect to the beam direction greater than $0.1 \mathrm{GeV} / c$. Energy clusters in the calorimeters were required to exceed minimum energy thresholds and those coming from noisy channels were excluded.

The detection efficiency for signal events depends on the mass of the scalar in the $\mathrm{S}^{0} \mathrm{Z}^{*}$ process. In the neutralino case it depends on the $\tilde{\chi}_{2}^{0}$ mass, on the mass difference $\Delta M^{0} \equiv\left(m_{\tilde{\chi}_{2}^{0}}-m_{\tilde{\chi}_{1}^{0}}\right)$ and on the $\tilde{\chi}_{2}^{0}$ decay modes. There is also a small dependence on the relative CP phase of the neutralinos, which affects the crosssections and angular distributions. Monte Carlo simulations were used in order to estimate the efficiency. For the simulation of the scalar production process $\mathrm{e}^{+} \mathrm{e}^{-} \rightarrow Z^{0} \rightarrow Z^{*} \mathrm{~S}^{0}$ the Monte Carlo generator PYTHIA [18] was used in its $\mathrm{e}^{+} \mathrm{e}^{-} \rightarrow \mathrm{Z}^{0} \rightarrow \mathrm{Z}^{*} \mathrm{H}^{0}$ generation mode. In the case of a visible scalar the decay modes were $\mathrm{Z}^{*} \rightarrow \nu \bar{\nu}$, $\mathrm{S}^{0} \rightarrow \mathrm{q} \overline{\mathrm{q}}$. Event samples were generated for scalar masses in the range $5-70 \mathrm{GeV} / c^{2}$. For the invisibly decaying $S^{0}$ boson, only $Z^{*}$ decays to $q \bar{q}$ were considered. Event samples were generated for invisible scalar masses in the range $1-70 \mathrm{GeV} / c^{2}$. Neutralino events were simulated using SUSYGEN [19], based on the formulæ of Ref. [20]. The processes considered were $\mathrm{e}^{+} \mathrm{e}^{-} \rightarrow \tilde{\chi}_{2}^{0} \tilde{\chi}_{1}^{0}$ where $\tilde{\chi}_{2}^{0} \rightarrow \tilde{\chi}_{1}^{0} \mathrm{q} \bar{q}$ and $\mathrm{e}^{+} \mathrm{e}^{-} \rightarrow \tilde{\chi}_{2}^{0} \tilde{\chi}_{2}^{0}$ where one of the neutralinos was forced to decay to $\tilde{\chi}_{1}^{0} q \bar{q}$ and no restriction was placed on the decay mode of the other. Events were generated corresponding to different points in the $\left(m_{\bar{\chi}_{2}^{0}}, m_{\tilde{\chi}_{1}^{0}}\right)$ plane and for both values of their relative $\mathrm{CP}$ phase $\left(\mathrm{CP}_{\tilde{\chi}_{2}^{0}} \mathrm{CP}_{\tilde{\chi}_{1}^{0}}= \pm 1\right)$. The regions where $\Delta M^{0} \leq 3 \mathrm{GeV} / c^{2}$ were not considered in the present analysis. The Lund fragmentation scheme does not reliably describe this region due to large non-perturbative QCD effects, and the experimental analysis was not designed to have good acceptance in this region.

To study the reaction $\mathrm{e}^{+} \mathrm{e}^{-} \rightarrow \tilde{\chi}_{2}^{0} \tilde{\chi}_{1}^{0}$, with $\tilde{\chi}_{2}^{0} \rightarrow \tilde{\chi}_{1}^{0} \gamma$, a simple generator was constructed. In the laboratory system this effectively produced an isotropic angular distribution of photons with a flat energy spectrum within the region allowed by kinematics. The known experimental acceptance and resolution for photons was then taken into account.

There are several possible sources of background to the signal topology, for which Monte Carlo simulations are needed:

- In the case of acoplanar events with large visible energy (corresponding to the cases of large $\Delta M^{0}$, heavy visible scalar or light invisible scalar), the most important background arises from multi-jet hadronic events originating from $Z^{0} \rightarrow q \bar{q}$ decays, in which one or more jet momenta are poorly determined. A sample of approximately 7.5 million $Z^{0} \rightarrow q \bar{q}$ events was generated using the JETSET [18] generator, with parameters tuned to OPAL data [21]. The most common reasons for poor determination of jet momenta are the production of heavy quarks decaying into energetic neutrinos, or the production of energetic neutrons or $\mathrm{K}_{\mathrm{L}}^{0}$ 's which could be poorly measured in the hadron calorimetry. A further sample of $Z^{0}$ decays was therefore generated using JETSET, applying a preselection to enhance such events before detailed simulation. This sample was equivalent to 33 million inclusive hadronic $Z^{0}$ decays.

- The process $\mathrm{e}^{+} \mathrm{e}^{-} \rightarrow \tau^{+} \tau^{-}(\gamma)$ may involve highly energetic neutrinos, yielding two acoplanar jets with large missing energy. Such events were generated with the KORALZ [22] event generator.

- In the cases where a visible scalar is light or an invisible scalar is heavy or when $\Delta M^{0}$ is small, the crents would be characterised by large missing energy. In this case, an important background can arise from twophoton scattering events in which energy escapes detection close to the beam direction. Such events were 
generated with PYTHIA [18], including both point-like $\gamma \gamma \rightarrow q \bar{q}$ processes and a vector meson dominance component.

- A further background to the present search arises from other four-fermion processes in which at least one of the fermions is a neutrino. Although the cross-section for these processes is small at centre-of-mass energies below the $\mathrm{W}^{+} \mathrm{W}^{-}$or $\mathrm{Z}^{0} \mathrm{Z}^{0}$ threshold, their event topology is similar to that of the signal. To simulate these events we used a generator based on helicity amplitude calculations, which takes into account all the relevant diagrams and their interference, and includes initial state radiation ${ }^{7}$.

- Finally in the case of single photon events, the main irreducible background comes from $\mathrm{e}^{+} \mathrm{e}^{-} \rightarrow \nu \bar{\nu} \gamma$ events. These events were produced with a generator based on Ref. [25].

Generated signal and background events were processed through the full simulation of the OPAL detector [26], and the same event analysis chain was applied to simulated events as to the data.

\section{Analysis}

\subsection{Acoplanar jets and mono-jets}

An accurate measurement of energy and of jet directions is crucial in order to distinguish between signal and background. The measurement of visible energy, missing momentum, visible mass and total transverse momentum of the events, and of jet angles, was performed by the method described in Ref. [16]. Fourmomentum vectors were formed for each track and calorimeter cluster, and then summed. The tracks were assigned the pion mass, unless identified as electrons or muons, and the calorimeter clusters were treated as massless particles. In order to reduce the effects of double counting, four-vectors based on the average expected energy deposition in the calorimeters for each charged track were then subtracted. This average energy deposition was parametrised as a function of track momentum and polar angle.

In most cases the signal consists of multi-jet events. However, if the invisible scalar is heavy, or both $\tilde{\chi}_{2}^{0}$ and $\widetilde{\chi}_{1}^{0}$ are light, the expected topology is often that of a mono-jet. In order to classify events as mono- or di-jets, each event was divided into two hemispheres by the plane orthogonal to the thrust direction. If there were no tracks and clusters passing the quality cuts in one of the hemispheres, the event was classified as a mono-jet. In the remaining events, classified as di-jets, the momentum sums in the two hemispheres were taken to define the two jets. In the following description, all cuts were applied to both classes unless otherwise specified.

The first group of cuts was applied as a preselection on the data:

- The numbers of charged tracks and of electromagnetic clusters were each required to be $\geq 5$. The cut was set to a low value in order to accept events where the mass difference between $\bar{\chi}_{2}^{0}$ and $\bar{\chi}_{1}^{0}$ is small, or the mass of the invisibly decaying scalar is large, leading to a small visible energy and low multiplicity.

- The fraction of tracks pointing to the interaction point was required to excecd $20 \%(50 \%)$ of the total number of tracks in the event in di-jet (mono-jet) events. This cut serves to eliminate background from offmomentum particles interacting in the beam pipe or low-multiplicity events containing hadronic interactions in the beam pipe or detector material.

- To reduce the number of hadronic $Z^{0}$ decays in the event sample, di-jet events were required to satisfy $\cos \theta_{\text {acol }}<0.98$ where $\pi-\theta_{\text {acol }}$ is the three-dimensional angle between the two jets. This cut rejected about $98 \%$ of the initial data sample.

- In order to ensure good energy containment, events with large energy flow near the beam direction were eliminated by the following cuts:

- The energies deposited in the forward (FD and SW) calorimeters were required to be less than $2 \mathrm{GeV}$ and $5 \mathrm{GeV}$ respectively.

\footnotetext{
${ }^{7}$ The calculation of the cross-section is based on the method described in a preprint by Murayama, Watanabe and Hagiwara [23]. The initial state radiation is implemented according to a formula in [24].
} 
Table 1

Numbers of events in data and Monte Carlo (scaled to the luminosity in the data) at various stages in the cuts, for mono-jet and di-jet events combined.

\begin{tabular}{lrrrrrr}
\hline Cuts & Data & $Z^{0} \rightarrow \mathrm{q} \bar{q}$ & Two-photon & $Z^{0} \rightarrow \tau^{+} \tau^{-}$ & Four-fermion & Total MC \\
\hline preselection & 34998 & 32561 & 3370 & 96 & 2.6 & 36030 \\
$p_{\text {T cut }}$ & 31536 & 32547 & 30 & 92 & 2.4 & 32671 \\
$\tau$ cuts & 31412 & 32518 & 30 & 18 & 2.3 & 32567 \\
non-spherical & 11461 & 12467 & 30 & 17 & 2.1 & 12516 \\
$\theta_{\text {acil }}$ and $\theta_{\text {acop }}$ & 20 & 16 & 0 & 2 & 1.5 & 19.5 \\
all cuts & 2 & 0.54 & 0 & 0.35 & 1.41 & 2.30 \\
\hline
\end{tabular}

The polar angle of the missing momentum vector was required to lie in the range $|\cos \theta|<0.94$.

- The forward energy flow divided by the total observed energy was required to be less than 10 . The forward energy flow [17] is the sum of the energies observed in forward and backward cones (defined by $|\cos \theta|>0.8$ ), weighted by $1 / \sin ^{2} \theta$.

- The $z$ component of the total event momentum was required to be less than $20 \mathrm{GeV} / c$.

After these preselection cuts, the number of events selected in data and the expectations from the Monte Carlo simulations are shown in Table 1.

In Fig. 1(a) we plot, for the di-jet events in the data, $p_{\mathrm{T}}$ against $M_{\text {vis }} / E_{\mathrm{c} . \mathrm{m} .}$, where $M_{\text {vis }}$ is the visible mass, $E_{\text {c.m. }}$ the centre-of-mass energy and $p_{\mathrm{T}}$ the total transverse momentum of the event, i.e. the magnitude of the vector sum of the transverse momenta. The clear cluster of events at low $p_{\mathrm{T}}$ and $M_{\text {vis }}$ is associated with twophoton interactions. This background was effectively eliminated by rejecting di-jet events having $M_{\mathrm{vis}} / E_{\mathrm{c} . \mathrm{m}}$. $<$ 0.27 and $p_{\mathrm{T}}<10 \mathrm{GeV} / c$. Fig. 1 (b) shows the $p_{\mathrm{T}}$ distribution for di-jet events having $M_{\text {vis }} / E_{\mathrm{c} . \mathrm{m} .}<0.27$ for data, in comparison with Monte Carlo. Mono-jet events were required to satisfy $p_{\mathrm{T}}>4 \mathrm{GeV} / c$. The value of $p_{\mathrm{T}}$ was calculated with and without the hadronic calorimeter information, and the minimum of the two values was taken. The value using the hadron calorimeter gave the minimum in only a few cases; however, this procedure was adopted in order to ensure optimal rejection of two-photon events. The observed and expected numbers of events after these cuts are again shown in Table 1.

The next group of cuts was designed to reject $Z^{0} \rightarrow \tau^{+} \tau^{-}$events:

- For events with less than 10 tracks the thrust value was required to be smaller than 0.95 .

- Some $\tau$-pairs show a mono-jet-like signature and therefore an additional requirement that the mass of the highest energy hemisphere exceed $2 \mathrm{GeV} / c^{2}$ was imposed.

- To remove radiative $\tau$ events, an additional requirement was imposed on events containing less than 10 charged tracks. Events containing an unassociated electromagnetic cluster of energy greater than $10 \mathrm{GeV}$ or a converted photon of energy greater than $6 \mathrm{GeV}$ were rejected, provided that the events were planar (specifically, the aplanarity $A<0.02$ where $A=\frac{3}{2} Q_{1}$ and $Q_{1}$ is the smallest eigenvalue of the sphericity tensor [27]). The observed and expected numbers of events after these cuts are again given in Table 1.

The remaining cuts are applied to di-jet events only. At this stage, the principal background is $Z^{0} \rightarrow q \bar{q}$ events. In order to search for acoplanar events within the remaining data (which are dominated by the back-to-back topology of the hadronic decays of the $Z^{0}$ ), we cut in the distributions of acollinearity $\left(\theta_{\text {acol }}\right)$ and acoplanarity ( $\theta_{\text {acop }}$, where $\pi-\theta_{\text {acop }}$ is the angle between the two jets in the $x-y$ plane). To ensure that the events have a non-spherical topology, so that $\theta_{\text {acol }}$ and $\theta_{\text {acop }}$ are well defined, events with thrust $<0.7$ were eliminated. In addition, events were rejected if the average hemisphere mass, $m_{\text {hemi }}^{\text {avg }}$, was greater than $20 \mathrm{GeV} / c^{2}$ or if the minimum of the hemisphere thrust, $T_{\text {hemj }}^{\mathrm{min}}$ (calculated in the centre-of-mass of each hemisphere separately) was less than 0.7. The observed and expected numbers of events after these cuts are compared in Table 1.

The cuts on $\theta_{\text {acol }}$ and $\theta_{\text {acop }}$ need to take account of the angular resolution, which depends on the topological nature of the events and also on the different geometrical regions of the detector. The remaining events were 

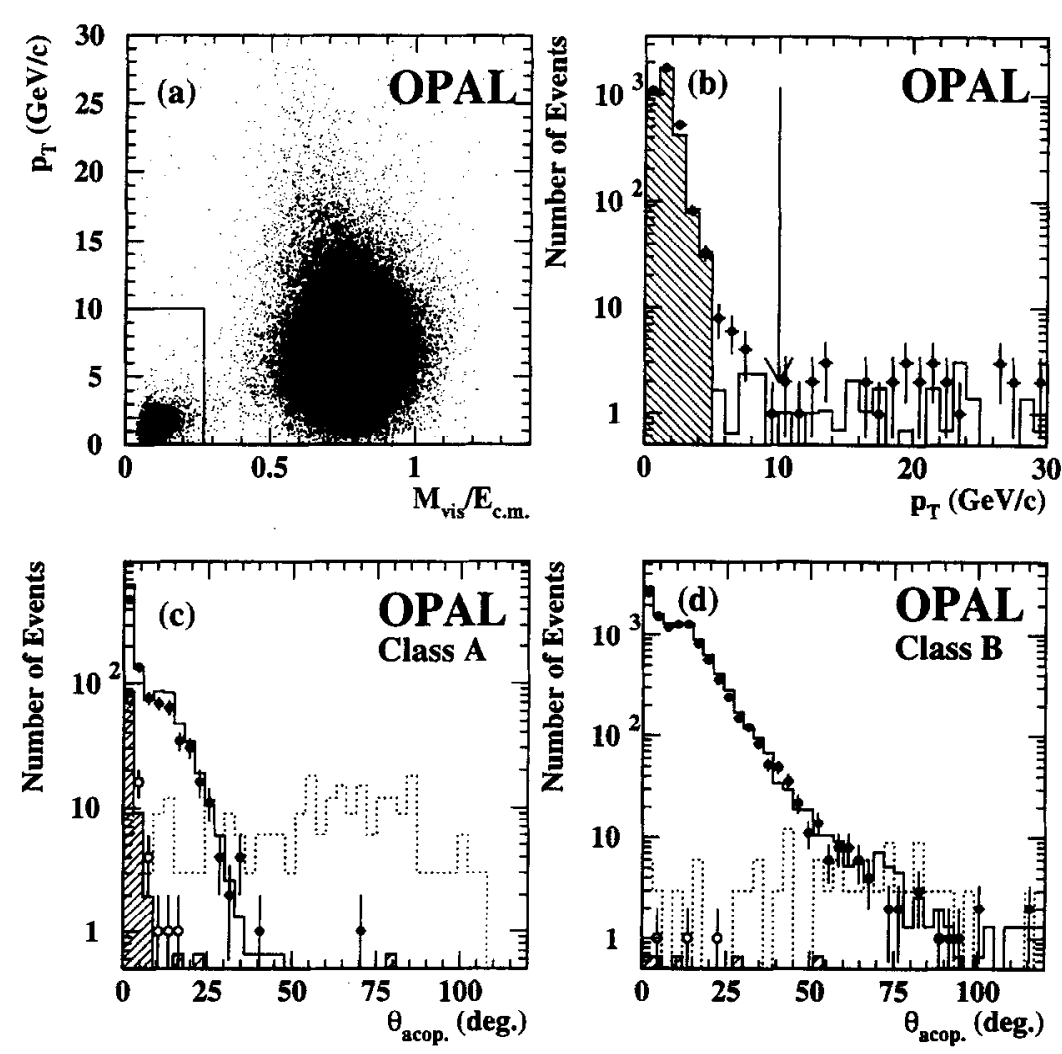

Fig. 1. (a) $p_{\mathrm{T}}$ vs. $M_{\text {vis }} / E_{\text {c.m. }}$ for di-jet data after the preselection cuts. The cut used to remove two-photon events is indicated. (b) Distribution of $p_{\mathrm{T}}$ for $M_{\mathrm{vis}} / E_{\mathrm{c} . \mathrm{m} .}<0.27$ for di-jet data, compared with Monte Carlo. The two-photon contribution is shown shaded. The cut applied is indicated by an arrow. (c) Distribution of $\theta_{\text {acop }}$ for class A for data (points) compared with Monte Carlo. The full points and open histogram are before the $\theta_{\text {acol }}$ cut is made, and the open points and hatched histogram are after the $\theta_{\text {acol }}$ cut is applied. The dotted histogram illustrates the expected distribution for neutralino pair production with masses of $\left(m_{\tilde{\chi}_{2}^{0}}, m_{\tilde{\chi}_{1}^{0}}\right)=(30,0) \mathrm{GeV} / \mathrm{c}^{2} .(\mathrm{d})$ Distribution of $\theta_{\text {acop }}$ for class B, using the same notation as (c).

Table 2

Class definitions and values of the cuts on the acollinearity and the acoplanarity angle. The barrel region is defined by $|\cos \theta|<0.71$ and the endcap region by $|\cos \theta| \geq 0.71$.

\begin{tabular}{llllll}
\hline Class & $T_{\text {hemi }}^{\text {min }}$ & $m_{\text {hemi }}^{\text {avg }}$ & Region & $\cos \theta_{\text {acol }}$ & $\cos \theta_{\text {acop }}$ \\
\hline A & $0.85-1.00$ & $0-11 \mathrm{GeV} / c^{2}$ & barrel & $<0.95$ & $<0.98$ \\
& & & endcap & $<0.90$ & $<0.95$ \\
B (excluding class A) & $0.70-1.00$ & $0-20 \mathrm{GeV} / c^{2}$ & both & $<0.70$ & $<0.5$ \\
\hline
\end{tabular}

divided into two classes $\mathrm{A}$ and $\mathrm{B}$, for which different acollinearity and acoplanarity cuts were applied. The class B events have broader jets, and thus the resolution on the angles is poorer. The cuts are given in Table 2. They are tighter than we used in Ref. [17], because Monte Carlo studies indicated that, with the present larger data sample, significant background would have been expected with the old cuts. The distributions of $\theta_{\text {acop }}$ for classes A and B are shown in Figs. 1 (c), (d) for data and Monte Carlo, and the observed and expected numbers of events after these cuts are again shown in Table 1.

The background events which survive the above cuts consist mainly of poorly measured multi-jet events, and thus the missing momentum generally lies close to one of the jets. These events are eliminated by demanding 
Table 3

Properties of the two events passing all cuts.

\begin{tabular}{|c|c|c|c|c|c|c|c|c|c|c|c|}
\hline \multirow[t]{2}{*}{ Run } & \multirow[t]{2}{*}{ Event } & \multicolumn{10}{|l|}{ Cut } \\
\hline & & Class & $\begin{array}{l}\text { Track } \\
\text { multiplicity }\end{array}$ & $\begin{array}{l}\text { Cluster } \\
\text { multiplicity }\end{array}$ & $\begin{array}{l}\text { Visible mass } \\
\left(\mathrm{GeV} / c^{2}\right)\end{array}$ & $\begin{array}{l}\text { Recoil mass } \\
\left(\mathrm{GeV} / c^{2}\right)\end{array}$ & $\begin{array}{l}\text { Event } p_{\mathrm{T}} \\
(\mathrm{GeV} / c)\end{array}$ & $\begin{array}{l}\text { Event } p_{z} \\
(\mathrm{GeV} / c)\end{array}$ & Thrust & $\theta_{\mathrm{acol}}$ & $\theta_{\text {acop }}$ \\
\hline 5436 & 107833 & di-jet & 8 & 12 & $24.8 \pm 3.0$ & $34.9 \pm 7.7$ & 32.1 & 2.2 & 0.84 & $92^{\circ}$ & $92^{\circ}$ \\
\hline 6543 & 214878 & mono-jet & 5 & 7 & $6.3 \pm 0.8$ & $78.5 \pm 1.3$ & 6.4 & 9.2 & 0.91 & - & - \\
\hline
\end{tabular}

that the sum of charged track and cluster energies in the calorimeters in a cone of half-angle $45^{\circ}$ around the missing momentum vector be less than $2 \mathrm{GeV}$. The observed and expected numbers of events after this final cut are presented in the last row of Table 1.

Two events from the full data sample survive all the selection cuts; their properties are summarised in Table 3 . The two observed candidates are consistent with the background expectation of $2.30 \pm 0.43$ events, consisting of $0.54 \pm 0.24$ events from hadronic decays of the $Z^{0}, 0.35 \pm 0.35$ events from $\tau^{+} \tau^{-}$, and $1.41 \pm 0.04$ events from four-fermion processes. Fewer than 0.6 events are expected with $M_{\text {vis }}$ exceeding $50 \mathrm{GeV} / c^{2}$. Although the remaining events exhibit no apparent deviation from expectations based on SM processes, both events are conservatively taken as signal candidates when interpreting the results of the search. Fig. 2(a) shows the distribution of $M_{\mathrm{vis}} / E_{\mathrm{c} . \mathrm{m}}$. for the surviving data and the background expectations. In Fig. 2(b) the Monte Carlo predictions for the signal after all cuts are shown for some typical cases: for a $60 \mathrm{GeV} / \mathrm{c}^{2}$ visible or a $70 \mathrm{GeV} / c^{2}$ invisible scalar particle or a pair of neutralinos with masses of $\left(m_{\tilde{\chi}_{2}^{0}}, m_{\tilde{\chi}_{1}^{0}}\right)=(30,0) \mathrm{GeV} / \mathrm{c}^{2}$. The predicted mass resolutions of these signals are taken into account when calculating limits.

\subsection{Single photon events}

The selection of single photon events followed closely the analysis of Ref. [28], which reported a measurement of the process $\mathrm{e}^{+} \mathrm{e}^{-} \rightarrow \nu \bar{\nu} \gamma$. The analysis was modified in order to concentrate on the search for high-energy photons. In particular, it was required that the scaled transverse energy of the photon, $x_{\mathrm{T}}=2 E \sin \theta / E_{\mathrm{c} . \mathrm{m} \text {. }}$ exceed 0.2 . The angular region considered was $|\cos \theta|<0.7$. A total of 31 single photon events was observed, consistent with the expectation from known processes of $27.1 \pm 2.1$, dominated by $\nu \bar{\nu} \gamma$ events. The observed spectrum of $x_{\mathrm{T}}$ is compared with $\nu \bar{\nu} \gamma$ Monte Carlo in Fig. 2(c). The expected number of events depends strongly on the photon energy. Since the expected energy spectrum from radiative neutralino decay is determined by the neutralino masses, we select an optimal cut on $x_{\mathrm{T}}$ (above $x_{\mathrm{T}}=0.2$ ) for each point in the $\left(m_{\tilde{\chi}_{3}^{0}}, m_{\tilde{\chi}_{1}^{0}}\right)$ plane so as to achieve maximal sensitivity to the existence of neutralinos. We parametrised the expected background from $\nu \bar{\nu} \gamma$ and evaluated the acceptance for radiative neutralino decay for each value of the $x_{\mathrm{T}}$ cut. The value of the $x_{\mathrm{T}}$ cut was chosen so that if there were no signal, and if observed events were produced at the rate expected for background events, the resulting upper limit on the signal process would be minimised. At each point in the $\left(m_{\tilde{X}_{2}^{0}}, m_{\tilde{\chi}_{1}^{0}}\right)$ plane the observed number of events is consistent with the expected background and thus $95 \%$ C.L. upper limits on the product branching ratio $\operatorname{BR}\left(Z^{0} \rightarrow \tilde{\chi}_{2}^{0} \tilde{\chi}_{1}^{0}\right) \operatorname{BR}\left(\tilde{\chi}_{2}^{0} \rightarrow \tilde{\chi}_{1}^{0} \gamma\right)$ were evaluated taking into account the expected background contribution.

\section{Efficiencies and systematic errors}

\section{1. signal efficiencies}

The detection efficiencies for scalar particles depend mainly on the mass and the decay modes. The efficiency for a heavy visible scalar produced in association with a $Z^{*}$ which decays into a pair of neutrinos is comparable 

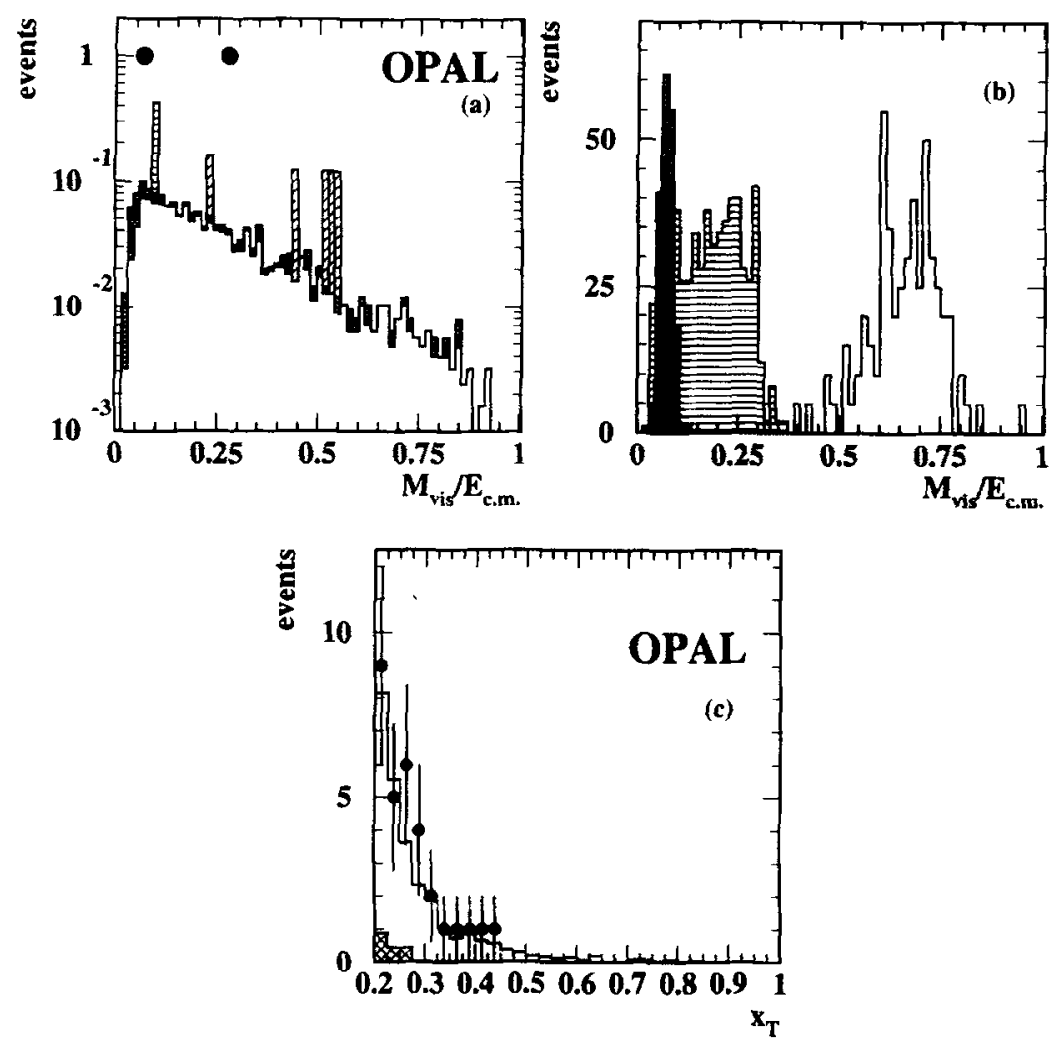

Fig. 2. (a) The scaled reconstructed mass, $M_{\text {vis }} / E_{\text {c.m. }}$, of the events in the mono-jet/di-jet search, after all cuts as described in the text. The events selected in data are shown as solid points, and the histogram shows backgrounds from multihadron (diagonal hatched), $\tau^{+} \tau^{-}$ (horizontal hatched) $\nu \bar{\nu} q \bar{q}$ (cross-hatched) and $e \nu \mathrm{q} \bar{q}^{\prime}$ (open). (b) The signals expected from Monte Carlo for a $60 \mathrm{GeV} / c^{2} v i s i b l e ~ s c a l a r$ (open), a $70 \mathrm{GeV} / c^{2}$ invisible scalar (cross-hatched) and a pair of neutralinos with masses of $\left(m_{\chi_{2}^{0}}, m_{\gamma_{1}^{0}}\right)=(30,0) \mathrm{GeV} / c^{2}($ horizontal

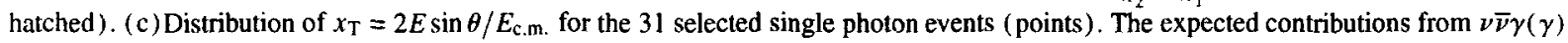
and $\ell^{\prime} \ell^{-} \gamma(\ell=\mu, \tau)$ are shown by the histograms; the non- $\nu \bar{\nu} \gamma(\gamma)$ contribution is hatched.

to that of a light invisible scalar produced in association with a $Z^{*}$ which decays to a pair of quarks. The efficiency for a visible scalar (not including the $Z^{*}$ branching ratio) reaches $50 \%$ at a scalar mass of around $40 \mathrm{GeV} / c^{2}$. It falls to $15 \%$ at $65 \mathrm{GeV} / c^{2}$ because the jets originating from a heavy scalar decay become more collinear, and falls to $30 \%$ for a $12 \mathrm{GeV} / c^{2}$ scalar owing to the low mass and $p_{\mathrm{T}}$ of the observed system. Likewise the efficiency for an invisible scalar was found to be about $26 \%$ for a very light invisible scalar, rising to $55 \%$ at $50 \mathrm{GeV} / c^{2}$, and falling to $22 \%$ at $70 \mathrm{GeV} / c^{2}$.

For neutralinos produced through the reaction $\tilde{X}_{2}^{0} \rightarrow \tilde{X}_{1}^{0} \mathrm{Z}^{*}$, with $\mathrm{Z}^{*} \rightarrow \mathrm{q} \overline{\mathrm{q}}$, the detection efficency at a given point in the $\left(m_{\tilde{\chi}_{2}^{0}}, m_{\tilde{X}_{1}^{0}}\right)$ plane depends on the value of $\mathrm{CP}_{\tilde{\chi}_{2}^{j}} \mathrm{CP}_{\tilde{X}_{1}^{0}}$. The lower of the two efficiencies was used for the limit calculation. The efficiencies were found to lie between $3 \%$ and $51 \%$ in the direct search for $\mathrm{e}^{+} \mathrm{e}^{-} \rightarrow \tilde{\chi}_{2}^{0} \tilde{\chi}_{1}^{0}$, and between $2 \%$ and $43 \%$ in the direct search for $\mathrm{e}^{+} \mathrm{e}^{-} \rightarrow \tilde{\chi}_{2}^{0} \tilde{\chi}_{2}^{0}$. The lower ends of these ranges of efficiencies correspond to the case of small $\Delta M^{0}$. In the case of radiative neutralino decay, $\tilde{\chi}_{2}^{0} \rightarrow \tilde{\chi}_{1}^{0} \gamma$, the efficiencies varied between 20 to $70 \%$ (within the fiducial region $|\cos \theta|<0.7$ ).

\subsection{Systematic errors}

The integrated luminosity was determined by counting multihadronic decays of the $Z^{0}$ and using the published OPAL hadronic cross-sections [29]. The systematic error from this procedure was $0.5 \%$, common to all 
channels.

The SM Higgs production cross-section serves as a convenient reference for the scalar particle search, and its uncertainty was taken to be $1.0 \%$ [30], taking into account the absolute cross-section and its dependence on the centre-of-mass energy.

The uncertainty associated with modelling of signal and background using Monte Carlo was evaluated taking account of Monte Carlo statistics, the effect of fragmentation, the uncertainty on $\alpha_{s}$ and the variation of selection cuts. The latter was accomplished by varying each of the selection cuts by approximately one standard deviation of the experimental resolution on the corresponding quantity, and determining the consequent change in the efficiency. It was found that the Monte Carlo statistical error dominated the uncertainties in all signal channels. The total Monte Carlo error varied between $1.8 \%$ and $5.0 \%$ for the visible scalar, between $3.1 \%$ and $6.1 \%$ for the invisible scalar, and between $3.0 \%$ and $18.0 \%$ for the neutralinos, depending on the masses.

The systematic and statistical errors were summed quadratically and subtracted from the signal selection efficiency.

\section{Results}

\subsection{Limits on scalar particle production rates and masses}

The production rate of a scalar particle of mass $m_{\mathrm{S}^{0}}$ in association with a $Z^{*}$, normalised to that of a SM Higgs boson of the same mass $m_{\mathrm{H}^{0}}$, is given by the ratio of the corresponding cross-sections. To set upper bounds on the normalised production rates of visible or invisible scalar particles, we used the expression

$$
\frac{B \cdot \sigma\left(\mathrm{e}^{+} \mathrm{e}^{-} \rightarrow \mathrm{S}^{0} \mathrm{Z}^{*}\right)}{\sigma\left(\mathrm{e}^{+} \mathrm{e}^{-} \rightarrow \mathrm{H}_{\mathrm{SM}}^{0} \mathrm{Z}^{*}\right)}=\frac{N\left(m_{\mathrm{S}^{0}}\right)}{\mathrm{BR}\left(\mathrm{Z}^{*} \rightarrow f \bar{f}\right) \cdot \sum_{E_{i}}\left[\epsilon\left(E_{i}, m_{\mathrm{S}^{0}}\right) \cdot \sigma\left(\mathrm{e}^{+} \mathrm{e}^{-} \rightarrow \mathrm{H}_{\mathrm{SM}}^{0} \mathrm{Z}^{*}\right)\left(E_{i}, m_{\mathrm{H}^{0}}\right) \cdot \mathcal{L}\left(E_{i}\right)\right]},
$$

where $\bar{f} \bar{f}=q \bar{q}$ or $f \bar{f}=\nu \bar{\nu}$ for an invisible or a visible scalar respectively, and $B$ is 1 or $B R\left(S^{0} \rightarrow q \bar{q}\right)$ respectively. The $\sigma$ 's are the production cross-sections, $\epsilon$ is the selection efficiency, and $\mathcal{L}$ is the integrated luminosity at an energy $E_{i}$. The function $N\left(m_{\mathrm{S}^{0}}\right)$ is the minimum number of expected events needed in order to set an upper bound at the $95 \%$ C.L. It was calculated using Poisson statistics and taking into account the mass resolution of the observed candidates [31].

The exclusion curves are shown in Fig. 3. Shoulders appear at masses corresponding to the candidate events passing the selection cuts. Degrading the mass resolution by up to $50 \%$ has only a minor effect on the curves. Fig. 3(a) shows that a visible scalar particle decaying entirely to quarks can be excluded for masses up to 60.8 $\mathrm{GeV} / \mathrm{c}^{2}$ at the $95 \%$ C.L. for a coupling strength to the $Z^{0}$ equivalent to that of the SM Higgs boson. A scalar with the expected decay branching ratios of the SM Higgs boson would be excluded up to $60.6 \mathrm{GeV} / c^{2}$. In a forthcoming publication, this result will be used together with other search channels to place limits on the SM Higgs boson. From Fig. 3(b) one can see that a massless invisible scalar particle is excluded if its production rate is larger than $2.8 \cdot 10^{-4}$ that of the SM Higgs Boson, while for the same production rate as that predicted for the SM Higgs, an invisible scalar is excluded if its mass is less than $67.5 \mathrm{GeV} / c^{2}$.

\subsection{Limits on neutralino production}

We obtain upper limits at the $95 \%$ C.L. on the $Z^{0} \rightarrow \tilde{\chi}_{2}^{0} \tilde{X}_{1}^{0}$ branching ratio, assuming specific decay modes. These limits do not depend on the details of SUSY models. The limit is computed as

$$
\frac{N\left(m_{\tilde{\chi}_{1}^{0}}, m_{\tilde{\chi}_{2}^{0}}\right)}{\sum_{E_{1}} \epsilon\left(m_{\tilde{\chi}_{1}^{0}}, m_{\tilde{\chi}_{2}^{0}}, E_{i}\right) \cdot \sigma\left(\mathrm{e}^{+} \mathrm{e}^{-} \rightarrow \mathrm{Z}^{0}\right)\left(E_{i}\right) \cdot \mathcal{L}\left(E_{i}\right)},
$$




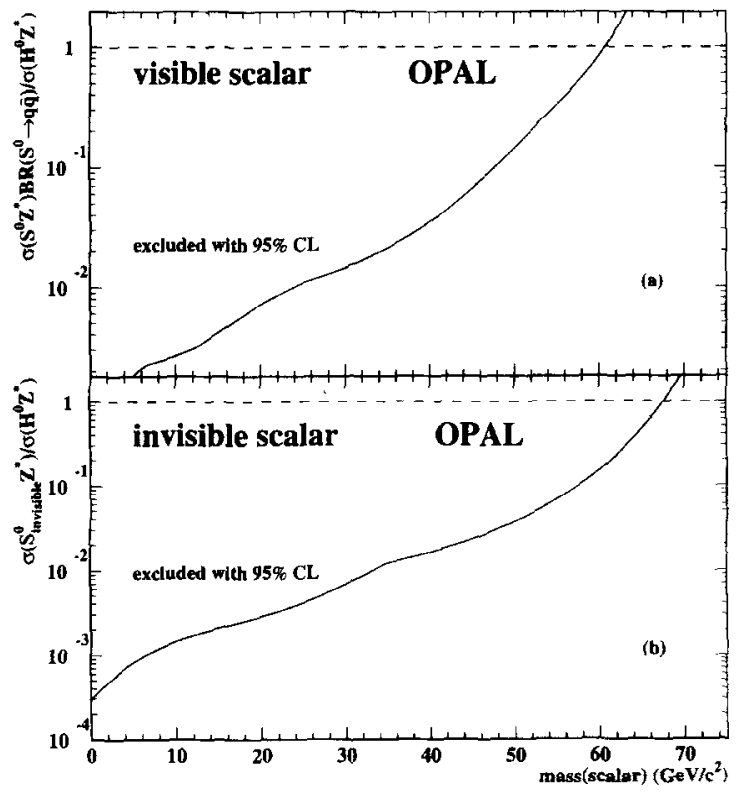

Fig. 3. The 95\% C.L. exclusion limits on the production rates of (a) a visible scalar decaying to quarks and (b) an invisibly decaying scalar.

where $N\left(m_{\tilde{X}_{1}^{0}}, m_{\tilde{\chi}_{2}^{0}}\right)$ is the minimum number of events needed to set a 95\% C.L. upper limit, $\epsilon\left(m_{\tilde{\chi}_{1}^{0}}, m_{\tilde{\chi}_{2}^{0}}, E_{i}\right)$ is the detection efficiency at energy $E_{i}$, and $\sigma\left(\mathrm{e}^{+} \mathrm{e}^{-} \rightarrow \mathbf{Z}^{0}\right)\left(E_{i}\right) \cdot \mathcal{L}\left(E_{i}\right)$ gives the number of $Z^{0}$ decays in the sample at energy $E_{i}$. The contours of the upper limits for the $Z^{0} \rightarrow \tilde{\chi}_{2}^{0} \tilde{\chi}_{1}^{0}$ process are shown for two cases: $\tilde{\chi}_{2}^{0} \rightarrow \tilde{\chi}_{1}^{0} Z^{*}$ with $100 \%$ branching fraction (Fig. 4(a)), or $\tilde{\chi}_{2}^{0} \rightarrow \tilde{\chi}_{1}^{0} \gamma$ with $100 \%$ branching fraction (Fig. 4(b)). The Standard Model branching fractions were used for the $Z^{*}$ decay. The dark region in Fig. 4(a) corresponds to $\Delta M^{0}<3 \mathrm{GeV} / c^{2}$, and was not considered in this analysis. The right-hand border of the triangular shape is the kinematic limit $m_{\tilde{\chi}_{2}^{0}}+m_{\tilde{\chi}_{1}^{0}}=m_{Z^{0}}$, where $m_{Z^{0}}$ is the $Z^{0}$ mass. One may infer, for example, from Fig. 4(a) that if $\Delta M^{0}>10 \mathrm{GeV} / c^{2}$ and the decay of the $\tilde{\chi}_{2}^{0}$ occurs purely via a $Z^{*}$ boson, then the branching fraction of $Z^{0} \rightarrow \tilde{\chi}_{2}^{0} \tilde{X}_{1}^{0}$ is smaller than $20 \cdot 10^{-6}$ at the $95 \%$ C.L. independent of the neutralino masses. Similarly from Fig. 4 (b) one can conclude that if $m_{\tilde{\chi}_{2}^{0}} / m_{\tilde{\chi}_{1}^{0}}>1.2$ and the $\tilde{\chi}_{2}^{0}$ decay is purely radiative, then the branching fraction of $Z^{0} \rightarrow \tilde{\chi}_{2}^{0} \tilde{X}_{1}^{0}$ is smaller than $100 \cdot 10^{-6}$ at the $95 \%$ C.L., independent of the neutralino masses.

The results of the above searches can be interpreted within the framework of the MSSM where the physics of the gaugino-higgsino sector of the theory is completely determined by three parameters: $M_{2}$, the mass of the supersymmetric partner of the W-boson at the weak scale when it is assumed that all the gauginos have a common mass at the grand-unification scale; $\mu$, the mass coupling strength between the two Higgs superfields, and $\tan \beta$, the ratio of the vacuum expectation values of the two Higgs doublets. The scanned regions of the parameter space were $0 \leq M_{2} \leq 1500 \mathrm{GeV} / c^{2}$ and $-400 \leq \mu \leq 400 \mathrm{GeV} / c^{2}$ for two values of $\tan \beta$ : $\tan \beta=1.5$, a small value interesting for infrared fixed-point predictions for the top quark [32], and $\tan \beta=35$, approximately equal to the ratio of the top quark mass to the $b$ quark mass favoured by Yukawa coupling unification at a large mass scale [33].

Two values were considered for the common mass scale of the sfermions, $m_{0}$ : either $m_{0}=1000 \mathrm{GeV} / c^{2}$ or the smallest $m_{0}$ consistent with present limits on the $\tilde{\ell}$ and $\tilde{\nu}$ masses, namely $m_{\tilde{\ell}}>45.0 \mathrm{GeV}$ and $m_{\tilde{\nu}}>$ $41.8 \mathrm{GeV}$ [34]. Although a small $m_{0}$ enhances neutralino production at energies close to the $Z^{0}$ pole owing to constructive interference between $s$-channel and $t$-channel exchange diagrams, the decay of $\tilde{\chi}_{2}^{0}$ to $\bar{\nu} \tilde{\nu}$, leading to unobserved final states, may be important for certain values of the other SUSY parameters, and thus may lead to less stringent limits. A careful consideration of both scenarios is therefore required.

Further constraints were imposed using experimental limits on the mass of the lightest chargino [35] and from limits on the excess in the width of the $Z^{0}(\Delta \Gamma<13.9 \mathrm{MeV}$ at the $95 \%$ C.L.) [36]. Points in the SUSY 

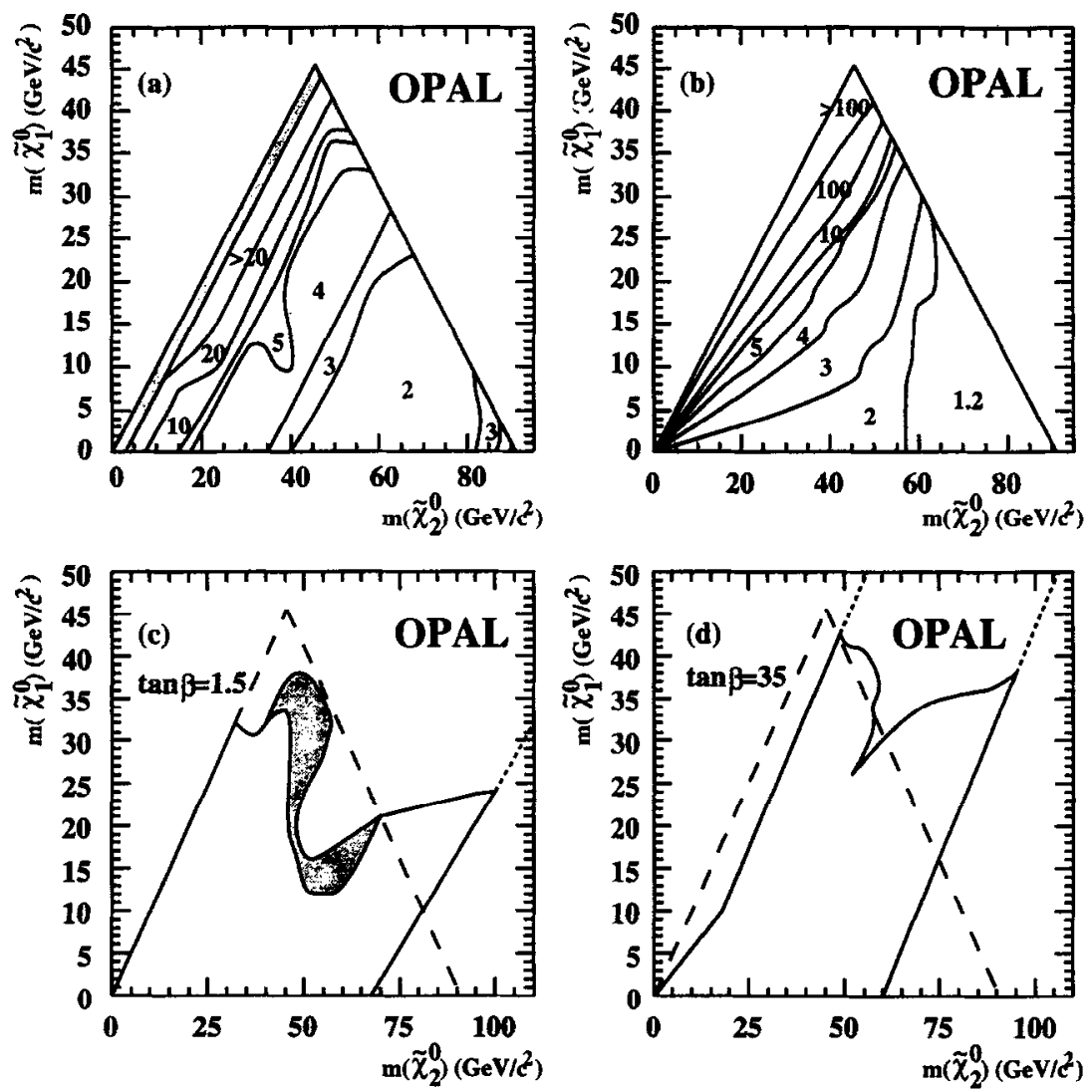

Fig. 4. Upper limits at $95 \%$ C.L. on product branching ratios for neutralino production: (a) $\operatorname{BR}\left(Z^{0} \rightarrow \tilde{\chi}_{2}^{0} \tilde{\chi}_{1}^{0}\right) \operatorname{BR}\left(\tilde{\chi}_{2}^{0} \rightarrow \tilde{\chi}_{1}^{0} Z^{*}\right)$. The curves show the contours corresponding to product branching ratios of $2,3,4,5,10,20 \cdot 10^{-6}$. (b) $\operatorname{BR}\left(Z^{0} \rightarrow \tilde{\chi}_{2}^{0} \tilde{\chi}_{1}^{0}\right) \operatorname{BR}\left(\tilde{\chi}_{2}^{0} \rightarrow \tilde{\chi}_{1}^{0} \gamma\right)$. The curves show the contours corresponding to product branching ratios of $1.2,2,3,4,5,10,100 \cdot 10^{-6}$. The results are interpreted in the framework of the MSSM in the $\left(m_{\tilde{\chi}_{2}^{0}}, m_{\tilde{\chi}_{1}^{0}}\right)$ plane for (c) $\tan \beta=1.5$ and (d) $\tan \beta=35$.0. The regions bounded by the solid lines are excluded at the $95 \%$ C.L. The regions outside the dotted lines are not accessible within the scanned parameter space. The dashed line on the left side of the triangle is the $\Delta M^{0}=0$ line which is accessible only via the $Z^{0}$ width constraint. The right dashed line is the kinematic boundary $\left(m_{\hat{X}_{2}^{0}}+m_{\tilde{X}_{1}^{0}}=m_{Z^{0}}\right)$. The region outside this line is also accessible only via the $Z^{0}$ width constraint. The shaded region in (c) is excluded for $m_{0}=1000 \mathrm{GeV} / c^{2}$, but not for a light $m_{0}$.

parameter space where either $m_{\tilde{\chi}_{1}^{+}}<47 \mathrm{GeV} / c^{2}$ provided $m_{\tilde{\chi}_{1}^{0}}<41 \mathrm{GeV} / c^{2}$ or $m_{\tilde{\chi}_{1}^{+}}<45.2 \mathrm{GeV} / c^{2}$, or where $\Gamma\left(Z^{0} \rightarrow \tilde{\chi}_{1}^{0} \tilde{\chi}_{1}^{0}\right)+\Gamma\left(Z^{0} \rightarrow \tilde{\chi}_{2}^{0} \tilde{\chi}_{1}^{0}\right)+\Gamma\left(Z^{0} \rightarrow \tilde{\chi}_{2}^{0} \tilde{\chi}_{2}^{0}\right)>\Delta \Gamma$, were excluded at the 95\% C.L. At each point in the MSSM parameter space, subject to these constraints, the expected decay rate of $Z^{0} \rightarrow \tilde{\chi}_{2}^{0} \tilde{\chi}_{1}^{0}$ was calculated. In regions of the parameter space where the $\tilde{\chi}_{2}^{0} \rightarrow \bar{\nu} \tilde{\nu}$ channel contributes, the predicted $Z^{0} \rightarrow \tilde{\chi}_{2}^{0} \tilde{\chi}_{1}^{0}$ branching ratio was reduced to account for this invisible final state. This expected branching ratio for visible $Z^{0} \rightarrow \tilde{\chi}_{2}^{0} \tilde{\chi}_{1}^{0}$ decays was then compared to the experimental limits obtained from the direct searches in both the $\tilde{\chi}_{2}^{0} \rightarrow \tilde{\chi}_{1}^{0} \gamma$ and the $\tilde{X}_{2}^{0} \rightarrow \tilde{X}_{1}^{0} Z^{*}$ channel. If the predicted $Z^{0} \rightarrow \tilde{X}_{2}^{0} \tilde{X}_{1}^{0}$ visible branching ratio was larger than both limits, the point was excluded. This method was adopted in order not to rely on predictions of the $\tilde{\chi}_{2}^{0} \rightarrow \tilde{\chi}_{1}^{0} \gamma$ branching ratio. Such predictions are rather uncertain, because the $\tilde{\chi}_{2}^{0} \rightarrow \tilde{\chi}_{1}^{0} \gamma$ decay proceeds via loop diagrams, and depends on assumptions about sfermion masses. The contribution of the direct search for $Z^{0} \rightarrow \tilde{\chi}_{2}^{0} \tilde{\chi}_{2}^{0}$ to the present exclusion proved to be minor.

The limits from the direct searches for $Z^{0} \rightarrow \tilde{\chi}_{2}^{0} \tilde{\chi}_{1}^{0}$ and $Z^{0} \rightarrow \tilde{\chi}_{2}^{0} \tilde{\chi}_{2}^{0}$ and the constraints from the $Z^{0}$ width and from the experimental bounds on the mass of the lightest chargino are combined to form exclusion regions in 

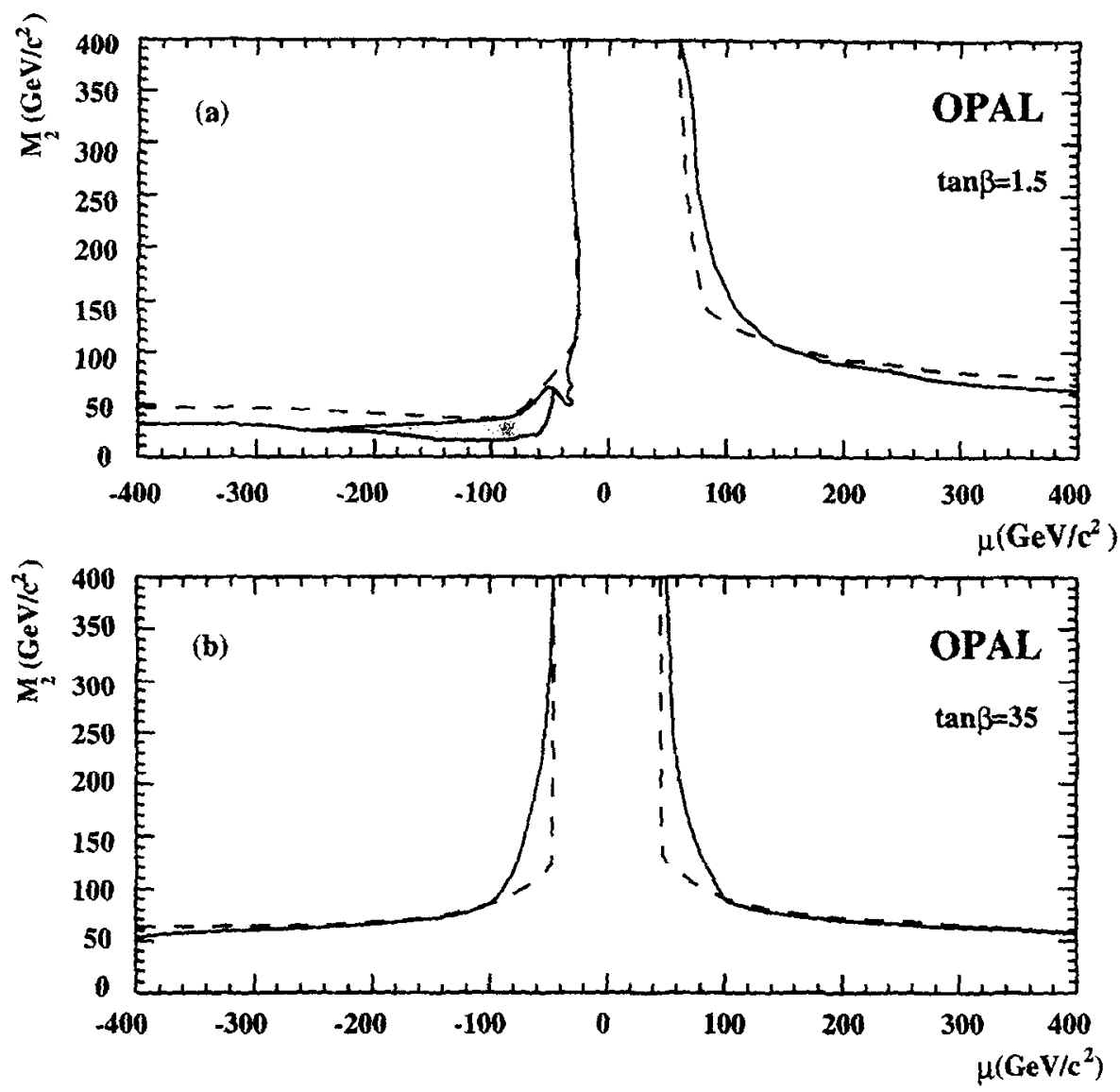

Fig. 5. The exclusion domain in the $\left(M_{2}, \mu\right)$ plane in the framework of the MSSM for (a) $\tan \beta=1.5$ and (b) $\tan \beta=35$. The regions bounded by the solid lines are excluded at the $95 \%$ C.L. The dashed lines represent the limits of the kinematically accessible region for $\tilde{\chi}_{2}^{(1} \tilde{\chi}_{1}^{0}$ production. The shaded region is excluded for $m_{i l}=1000 \mathrm{GeV} / c^{2}$, but not for a light $m_{0}$.

the $\left(m_{\tilde{\chi}_{2}^{0}}, m_{\tilde{\chi}^{0}}\right)$ and $\left(\mu, M_{2}\right)$ planes, as shown in Figs. 4 and 5 . The exclusion domain in the $\left(m_{\tilde{\chi}_{2}^{0}}, m_{\tilde{\chi}_{1}^{0}}\right)$ plane is shown in Fig. $4(\mathrm{c})$ for $\tan \beta=1.5$ and $4(\mathrm{~d})$ for $\tan \beta=35$. The regions bounded by the solid lines are excluded at the $95 \%$ C.L. The regions outside the dotted lines are not accessible within the scanned parameter space. The dashed line on the left side of each triangle is the $\Delta M^{0}=0$ line, which is accessible only via the $\mathrm{Z}^{0}$ width constraint. The right dashed line is the kinematic limit for $\tilde{\chi}_{2}^{0} \tilde{\chi}_{1}^{0}$ production $\left(m_{\tilde{\chi}_{2}^{0}}+m_{\tilde{\chi}_{1}^{0}}=m_{Z^{0}}\right)$. Sensitivity to the region beyond this line comes only from the $Z^{0}$ width constraints. The following lower limits can be set on the neutralino masses: $m_{\tilde{X}_{1}^{0}}>12.5 \mathrm{GeV} / c^{2}$ and $m_{\tilde{X}_{2}^{0}}>33.0 \mathrm{GeV} / c^{2}$ for $\tan \beta=1.5$, and $m_{\tilde{X}_{1}^{0}}>26.0 \mathrm{GeV} / c^{2}$ and $m_{\tilde{\chi}_{2}^{0}}>51.5 \mathrm{GeV} / c^{2}$ for $\tan \beta=35$. Taking $m_{0}=1000 \mathrm{GeV} / c^{2}$, the lower limit on the lightest neutralino becomes $m_{\tilde{\chi}_{1}^{0}}>16.3 \mathrm{GeV} / c^{2}$ for $\tan \beta=1.5$.

The exclusion domain in the $\left(\mu, M_{2}\right)$ plane is shown in Fig. $5(\mathrm{a})$ for $\tan \beta=1.5$ and in Fig. 5(b) for $\tan \beta=35$. The region bounded by the solid lines is excluded at the $95 \%$ C.L. Note that the measurement of the $Z^{0}$ width allows the limits to be extended beyond the kinematically accessible region for $\tilde{\chi}_{2}^{0} \tilde{\chi}_{1}^{0}$ production (represented by the dashed lines). Finally it should be noted that the case of a smaller $\tan \beta$ was also studied. We find that if $1<\tan \beta<1.3$, the $Z^{0}$ width cannot exclude neutralinos with a small $\Delta M^{0}$ and one cannot set a limit on the masses of neutralinos.

These results update a previous OPAL publication [37] and can be compared with other LEP publications [38]. 


\section{Summary and conclusions}

We have analysed a data sample corresponding to an integrated luminosity of $160 \mathrm{pb}^{-1}$, collected around the $\mathrm{Z}^{0}$ peak with the OPAL detector, to search for acoplanar jets or mono-jets motivated by supersymmetry and other theories. Two events remained after the selection, consistent with the background expectation of $2.30 \pm 0.43$ events from hadronic, $\boldsymbol{\tau}^{+} \tau^{-}$and four-fermion processes.

Limits have been placed on the production, in association with a $\mathrm{Z}^{*}$, of invisible scalar particles and of visible scalars decaying to quarks. A massless invisible scalar is excluded if its production rate is more than $2.8 \cdot 10^{-4}$ that of the SM Higgs boson. A scalar particle whose production rate is the same as the SM Higgs boson can be excluded at the $95 \%$ C.L. for masses up to $67.5 \mathrm{GeV} / c^{2}$ (or $60.8 \mathrm{GeV} / c^{2}$ ) if it decays entirely into invisible final states (or entirely into quarks).

In placing limits on neutralino production, single photon events and information on the $Z^{0}$ width are also included in the analysis. At 95\% C.L., lower limits on the lightest and next to lightest neutralino masses within the Minimal Supersymmetric Standard Model are set at $12.5 \mathrm{GeV} / c^{2}$ and $33.0 \mathrm{GeV} / c^{2}$ respectively for $\tan \beta>1.5$. Taking $m_{0}=1000 \mathrm{GeV} / c^{2}$, the lower limit on the lightest neutralino becomes $m_{\tilde{\chi}_{1}^{0}}>16.3 \mathrm{GeV} / c^{2}$.

\section{Acknowledgements}

It is a pleasure to thank the SL Division for the efficient operation of the LEP accelerator, the precise information on the absolute energy, and their continuing close cooperation with our experimental group. In addition to the support staff at our own institutions we are pleased to acknowledge the

Department of Energy, USA,

National Science Foundation, USA,

Particle Physics and Astronomy Research Council, UK,

Natural Sciences and Engineering Research Council, Canada,

Israel Ministry of Science,

Israel Science Foundation, administered by the Israel Academy of Science and Humanities,

Minerva Gesellschaft,

Japanese Ministry of Education, Science and Culture (the Monbusho) and a grant under the Monbusho International Science Research Program,

German Israeli Bi-national Science Foundation (GIF),

Direction des Sciences de la Matière du Commissariat à l'Energie Atomique, France,

Bundesministerium für Bildung, Wissenschaft, Forschung und Technologie, Germany,

National Research Council of Canada,

Hungarian Foundation for Scientific Research, OTKA T-016660, and OTKA F-015089.

\section{References}

| 1 | S.L. Glashow, J. lliopoulos and L. Maiani, Phys. Rev. D 2 (1970) 1285; S. Weinberg, Phys. Rev. Lett. 19 (1967) 1264;

A. Salam, Elementary Particle Theory, ed. N. Svartholm (Almquist and Wiksells, Stockholm, 1968) 367.

12) P.W. Higgs, Phys. Lett. 12 (1964) 132;

F. Englert and R. Brout, Phys. Rev. Lett. 13 (1964) 321;

G.S. Guralnik, C.R. Hagen, and T.W.B. Kibble, Phys. Rev. Lett. 13 (1964) 585.

[3] H.E. Haber and Y. Nir, Nucl. Phys B 335 (1990) 363.

14| R. Barbieri et al., Z. Physics at LEP1, CERN 89-08 (1989) Vol. 2, 121;

J.M. Frere, G.L. Kane, Nucl. Phys. B 223 (1983) 331;

J. Ellis et al., Phys. Lett. B 123 (1983) 436; B 127 (1983) 233.

15| P. Fayet, Unification of the Fundamental Particle Interactions, Plenum Press (1980) p. 587. 
$16 \mid$ J. Wess and B. Zumino, Nucl. Phys. B 70 (1974) 39;

H. Nilles, Phys. Rep. 10 (1984) 1:

R. Barbieri, Riv. Nuovo Cimento 11 (1988).

$17 \mid$ H.E. Haber and D. Wyler, Nucl. Phys. B 323 (1989) 267;

S. Ambrosanio and B. Mele, hep-ph/9508237, submitted to Phys. Rev. D.

$18 \mid$ Y. Chikashige, R.N. Mohapatra and R.D. Peccei, Phys. Lett. B 98 (1980) 265.

19] D.B. Reiss. Phys. Lett. B 115 (1982) 217: F. Wilczek, Phys. Rev. Lett. 49 (1982) 1549;

G. Gelmini and M. Roncadelli, Phys. Lett. B 99 (1981) 411;

A.S. Joshipura and S.D. Rindani, Phys. Rev. D 46 (1992) 3000.

$110 \mid$ A.S. Joshipura, J.W.F. Valle, Nucl. Phys. B 397 (1993) 105.

111 J J.C. Romao, F. de Campos and J.W.F. Valle, Phys. Lett. B 292 (1992) 329.

112 | J.W.F. Valle and J.T. Peltoniemi, Massive Neutrinos and Electroweak Baryogenesis, gls0788 in parallel session pa-04 Beyond the Standard Model. Proc. 27th Intern. Conf. on High Energy Physics, Glasgow (United Kingdom), July 20-27, 1994.

113] R.E. Schrock and M. Suzuki, Phys. Lett. B 110 (1982) 250;

L.F. Li, Y. Liu and L. Wolfenstein. Phys. Lett. B 159 (1985) 45;

E.D. Carlson and L.B. Hall, Phys. Rev. D 40 (1985) 3187:

G. Jungman and M.A. Luty, Nucl. Phys. B 361 (1991) 24;

A.S. Inshipura and S.D. Rindani, Phys. Rev. Lett. 69 (1992) 3269;

J.D. Bjorken, Invited Talk at the Symposium on the SSC Laboratory, Corpus Christi, Texas, October 1991, SLAC-PUB-5673 (1991).

114| OPAL Collaboration, K. Ahmet et al., Nucl. Instrum. Methods A 305 (1991) 275.

| $15 \mid$ B.E. Anderson et al., IEEE Transactions on Nuclcar Science 41 (1994) 845.

[16] OPAL Collaboration, M.Z. Akrawy et al., Phys. Lett. B 253 (1991) 511.

| 17 | OPAL Collaboration, R. Akers et al., Phys. Lett. B 327 (1994) 397.

1181 JETSET, Version 7.4 and PYTHIA, Version 5.7; T. Sjöstrand, Comp. Phys. Comm. 82 (1994) 74.

[19] SUSYGEN: An event generator for supersymmetric particle production, S. Katsanevas and S. Melachronios, to be published in the LEP200 workshop report, CERN 1996.

201 A. Bart, H. Frass and W. Majeroto, Nucl. Phys. B 278 (1986) 1.

121| OPAL Collaboration, P. Acton et al., 7. Phys. C 58 (1993) 387;

OPAL Collaboration, G. Alexander et al., CERN-PPE/95-126 Z. Phys. C, to be published.

|22| KORALZ, Version 4.0, S. Jadach et al., Comp. Phys. Comm. 66 (1991) 276.

|23] H. Murayama, I. Watanabe and K. Hagiwara, KEK Report 91-11 (1991).

124| F.A. Berends, R. Pittau and R. Kleiss, Nucl. Phys. B 426 (1994) 344.

125| F. A. Berends et al., Nucl. Phys. B 301 (1988) 583;

R. Miquel, C. Mana and M. Martinez, L. Mhys. C 48 (1990) 309.

126| J. Allison et al., Nucl. Instrum. Methods A 317 (1992) 47: GEANT Detector Description and Simulation Tool, CERN Program Library Long Writeup W5013 (1993).

127| S.L. Wu and G. Zobernig, Z. Phys. C 2 (1979) 107.

|28| OPAL Collaboration, R. Akers et al., Z. Phys. C 65 (1995) 47.

129| OPAL Collaboration, G. Alexander et al., Z. Phys. C 52 (1991) 175 ;

OPAL Collaboration, P.D. Acton et al., Z. Phys. C 58 (1993) 219;

OPAL Collaboration, R. Akers et al.. Z. Phys. C 61 (1994) 19.

|30| E. Gross, B. Kniehl and G. Wolf. Z. Phys. C 63 (1994) 417; C 66 (1995) 321 (E).

$131 \mid$ E. Gross and P. Yepes, Intern. J. Mod. Phys. A 8 (1993) 407.

|32| M. Carena and C.E.M. Wagner, Nucl. Phys B 452 (1995) 45.

$133 \mid$ J. Ellis, S. Kelley, and D.V. Nanopoulos, Phys. Lett. B 287 (1992) 95.

134| Particle Data Group, Phys. Rev. D 50 (1994) 1173.

1.35) ALEPH Collaboration, D. Decamp et al., Phys. Rep. 216 (1992) 253.

|36| D. Schaile, Fortschr. Phys. 42 (1994) 429.

1.37| OPAL Collaboration, M.Z. Akrawy et al., Phys. Lett. B 248 (1990) 211.

| 38 | 1.3 Collaboration, M. Acciarri et al.. Phys. Lett. B 350 (1995) 109;

ALEPH Collaboration, D. Decamp et al., Phys. Lett. B 244 (1990) 541; Phys. Rep. 216 (1992) 253;

DELPHI Collaboration, P. Abreu et al., Phys. Lett. B 247 (1990) 157. 